\title{
UNIDADES DE CONSERVAÇÃO: UMA RECONEXÃO COM A NATUREZA, PÓS-COVID-19
}

\author{
Marta Regina da Silva-Melo ${ }^{1}$ \\ Gleidson André Pereira de Melo² \\ Neiva Maria Robaldo Guedes ${ }^{3}$
}

Resumo: Esta pesquisa tem como objetivo analisar a importância das Unidades de Conservação como espaços de reconexão com a natureza, pósCOVID-19. Os procedimentos que orientaram o estudo contribuíram com a fase descritiva dos fenômenos e sua relação com a necessidade de aprimorar tendências biofílicas. A partir de um amostra aleatória não-probabilista, os resultados apontaram que ambientes naturais são capazes de proporcionar sensação de bem-estar, após a pandemia do novo coronavírus. Diante de um novo "normal," as Unidades de Conservação se notabilizam como espaços naturais importantes para amenizarem os efeitos da ansiedade e estresse causados pela COVID-19.

Palavras-chave: Biofilia; Áreas Protegidas; Ambiente Restauradores.

Abstract: This research aims to analyze the importance of Conservation Units as spaces for reconnecting with nature, after COVID-19. The procedures that guided the study contributed to the descriptive phase of the phenomena and their relationship to the need to improve biophilic trends. From a nonprobabilistic random sample, the results showed that natural environments are capable of providing a sense of well-being after the new coronavirus pandemic. Faced with a new "normal," the Conservation Units stand out as important natural spaces to decreasing the effects of anxiety and stress caused by COVID-19.

Keywords: Biophilic; Protected Areas; Restorative Environments.

\footnotetext{
1 Universidade Anhanguera-Uniderp. E-mail: martamelors@gmail.com. Link para o Lattes: http://lattes.cnpq.br/8192425520595557

2Instituto Federal de Mato Grosso do Sul. E-mail: gandmelo@gmail.com. Link para o Lattes: http://lattes.cnpq.br/1370968666001171

3Universidade Anhanguera-Uniderp. E-mail: guedesneiva@gmail.com. Link para o Lattes: http://lattes.cnpq.br/7358580565148346
} 


\section{Introdução}

As Unidades de Conservação (UCs), representadas por Parques, Reservas Particulares do Patrimônio Natural, Estradas-Parque, Áreas de Proteção Ambiental, Reservas do Desenvolvimento Sustentável, entre outras, constituem uma das mais eficientes estratégias para a proteção da diversidade biológica brasileira. Essas áreas cumprem uma série de funções, e dentre essas, a regulação da qualidade de água para consumo, fertilidade dos solos, equilíbrio climático, manutenção da qualidade do ar, base para produção de medicamentos, áreas verdes para lazer, educação, cultura, ecoturismo e reflexão espiritual (FIGGIS, 2015; MARTINS, 2018; LANZAS, 2019). Com efeito, as UCs são espaços que têm relação direta com o conceito de ambientes restauradores (ULRICH, 1983). Isso ocorre em virtude de uma relação positiva com ambientes naturais que favorecem o bem-estar humano $\mathrm{e}$ podem propiciar a redução do estresse e os desgastes cotidianos

A hipótese da biofilia, criada por Wilson (1984), ressalta que o ser humano apresenta necessidades profundas de contato com a natureza. Isso ocorre em razão de uma necessidade biológica, visto que nossa composição genética se estruturou em função de convivência com ambientes naturais, e não em ambientes artificiais. Essa conexão com a natureza pode ser compreendida como uma condição primária e fundamental da nossa espécie (WILSON,1984). Entretanto, alguns estudos apontam que nem sempre o laço genético é o suficiente. Todavia, requer algum aprendizado cultural e vivências relacionadas com a natureza para a otimização das tendências de biofilia (KELLERT, 2012).

O modo de vida, perspectivas e as ações da sociedade contemporânea, podem ter influenciado a maneira de se relacionar com a natureza, ampliando o distanciamento e perdas das relações com a natureza. A diminuição do contato com o ambiente natural pode ajudar a explicar a ligação entre urbanização e doença mental, estimulada por um padrão desadaptativo que aumenta a depressão e outros transtornos mentais (BRATMAN, et al. 2015).

Quando o ser humano não cultiva o contato com a natureza, é possível manifestar transtorno de déficit de natureza (LOUV, 2016), que pode estar relacionado às consequências negativas para a saúde, ocasionadas pela falta de relacionamento com ambientes naturais, para quem vive nos centros urbanos. Estar em sintonia com a natureza, além de proporcionar benefícios para a saúde do corpo, pode aumentar nossa sensibilidade quanto à importância de conservação e cuidados com o ambiente natural. Pressupõe-se que qualquer tipo de ligação estabelecida com a natureza, nas quais as pessoas possam estar engajadas, possibilitará o aprimoramento dos aspectos cognitivos (SCHERTZ; BERMAN, 2019), afetivos e socioculturais.

Com o advento da pandemia da COVID-19, o governo brasileiro tornou pública a Lei $\mathrm{n}^{\circ}$ 13.979/2020, que dispõe sobre as medidas para o enfrentamento da emergência de saúde pública de importância internacional, decorrente do novo coronavírus (MACÉDO et. al., 2020). A preocupação 
desencadeada em consequência da COVID-19 tem implicado em problemas de saúde mental, acentuando instabilidades devido ao cenário mundial de fragilidade e incertezas (MACÊDO et. al., 2020; RAJKUMAR, 2020), dessa forma, o medo da pandemia reforça o estado de ansiedade como fonte da ameaça ao bem-estar coletivo e individual (FUREDI, 2013), e, na atualidade, a paranoia e o pânico (HO; CHEE; HO, 2020)

$\mathrm{O}$ contato com ambiente natural pode minimizar diferentes males emocionais e físicos que enfrentamos. Dessa feita, estudos científicos revelam que a exposição direta à natureza é essencial para a saúde física e emocional (VAN DEN BERG et al., 2012; MOGHADAM, SINGH, YAHYA, 2015; LOUV, 2016; HUGHES et al., 2019). Além disso, a vida ao ar livre é um dos melhores antídotos contra a intoxicação digital (BECKER et al., 2019).

Dessa forma, a importância deste estudo consiste na função de que as Unidades de Conservação desempenham um papel importante como ambientes restauradores e produzem efeitos benéficos para o bem-estar humano, diante da necessidade de um novo "normal", e, portanto, reconexão é a palavra-chave para quem busca a integração com o meio natural.

Esta pesquisa tem como objetivo analisar a importância das Unidades de Conservação como espaços de reconexão com a natureza e bem-estar humano, pós-COVID-19. Especificamente, identificar os benefícios do contato com o ambiente natural, destacar os efeitos restauradores das Unidades de Conservação para o bem-estar humano e relacionar práticas capazes de reconectar pessoas à natureza.

\section{Metodologia}

Os procedimentos metodológicos que orientaram a elaboração desta pesquisa envolveram consultas em fontes especializadas sobre a temática e a fundamentação teórica referentes aos aspectos analisados. Ainda neste sentido, ocorreu a fase descritiva das características e dos fenômenos, a fim de classificar, explicar e interpretar os fatos, bem como, registrar, analisar e correlacionar sua relação com variáveis sociais, ambientais e bem-estar, com base em fontes de informação indiretas (pesquisa documental, bibliográfica e digital), para fim de justificar os temas envolvidos: áreas naturais, Unidades de Conservação, bem-estar humano, efeitos restauradores e conexão com a natureza, pós COVID-19.

Foram considerados aspectos apontados na hipótese da biofilia apresentada por Edward Wilson (1984); e no que foi proposto pelo ecologista social Stephen Kellert (2012), onde afirma que o ser humano necessita estabelecer um aprendizado com a natureza para intensificar a tendência de biofilia. Bem como, características sobre o transtorno de déficit de natureza, termo criado por Richard Louv (2016). Por essas razões foi elaborado um diagrama (Figura 1), com conceitos-chave desses estudos, e por permitir uma visão sintética e conjunta das inter-relações com o ambiente natural. 


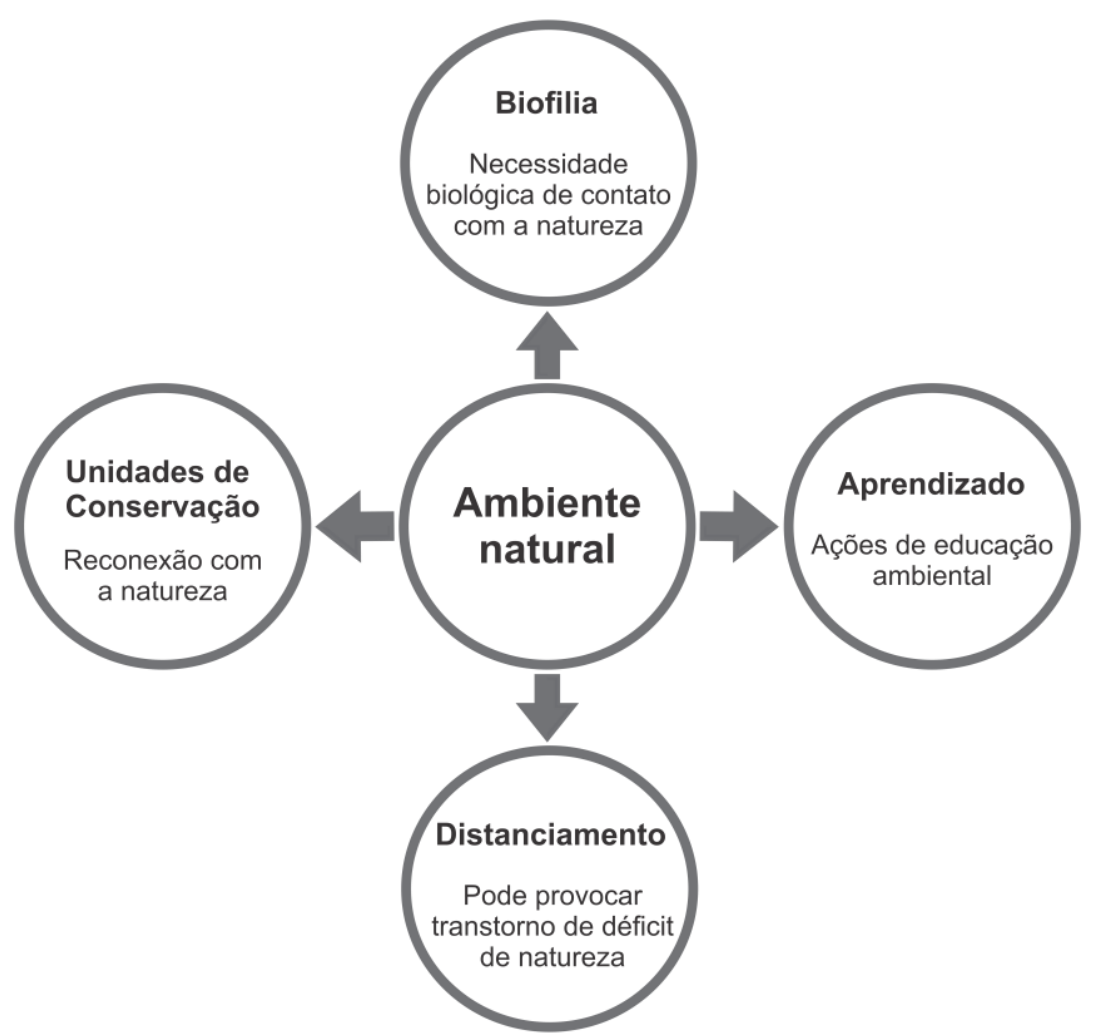

Figura 1: Diagrama das inter-relações com o ambiente natural.

Fonte: Elaborado pelos autores.

Além disso, foi realizado um levantamento por meio de um instrumento de coleta de dados, para uma amostra aleatória não probabilista $(n=354)$, nas diferentes regiões do território brasileiro. Com a finalidade de reunir dados sobre os ambientes nos quais as pessoas acreditam que são capazes de proporcionar sensação de bem-estar, após a pandemia da COVID-19.

\section{Resultados e Discussões}

É apropriado sinalizar que a preocupação em consequência da COVID19 tem intensificado problemas emocionais, situação que afetou as pessoas nos diferentes continentes do globo. Pois, o isolamento social forçado fez com que os casos de estresse e ansiedade mais que dobrassem (PANCANI et al., 2020), e no Brasil não pôde ser diferente.

Nesse contexto, em um momento tão complexo ocasionado pela pandemia da COVID-19, quando diferentes pesquisas científicas sinalizam que o contato com a natureza oportuniza às pessoas diversos benefícios (Quadro 1), há uma compreensão de que as Unidades de Conservação, com cobertura significativa de parcela do território nacional, podem propiciar efeitos restauradores para o bem-estar humano. 
Quadro 1: Benefícios proporcionados às pessoas pelo contato com a natureza.

\begin{tabular}{|l|l|}
\hline \multicolumn{1}{|c|}{ Descrição dos benefícios } & \multicolumn{1}{|c|}{ Autores } \\
\hline $\begin{array}{l}\text { O envolvimento com o ambiente natural reforça nas pessoas a } \\
\text { conexão e identidade afetiva sobre a proteção ambiental. }\end{array}$ & Hinds, Sparks (2007) \\
\hline $\begin{array}{l}\text { A conexão com a natureza pode ser considerada uma atitude } \\
\text { baseada em valores. }\end{array}$ & $\begin{array}{l}\text { Brügger, Kaiser, Roczen } \\
(2011)\end{array}$ \\
\hline $\begin{array}{l}\text { Atividades na natureza proporcionam múltiplos benefícios para } \\
\text { o bem-estar e restauração humana. }\end{array}$ & $\begin{array}{l}\text { Keniger, Gaston, Irvine, Fuller } \\
(2013) .\end{array}$ \\
\hline $\begin{array}{l}\text { A natureza tem sido a fonte do bem-estar físico e psicológico } \\
\text { da saúde humana, desde o início de sua existência. }\end{array}$ & $\begin{array}{l}\text { Moghadam, Singh, Yahya } \\
(2015)\end{array}$ \\
\hline $\begin{array}{l}\text { O conato com ambientes naturais é um remédio sem } \\
\text { contraindicação, e a "Vitamina N de Natureza" é uma receita } \\
\text { completa para se conectar com o poder e a alegria do mundo } \\
\text { natural. }\end{array}$ & Louv (2016) \\
\hline $\begin{array}{l}\text { Observar os elementos da natureza pode proporcionar } \\
\text { aumentos sustentados na conexão das pessoas com o } \\
\text { ambiente natural à melhoria da saúde psicológica. }\end{array}$ & Richardson, Sheffield (2017) \\
\hline $\begin{array}{l}\text { A exposição aos ambientes naturais melhora o bem-estar, } \\
\text { sugerindo que interagir com a natureza pode ser uma via pela } \\
\text { qual os indivíduos podem alcançar e manter um duradouro } \\
\text { senso de felicidade. }\end{array}$ & McMahan (2018) \\
\hline $\begin{array}{l}\text { O contato regular com a natureza melhora a saúde, } \\
\text { comportamentos sociais ao longo da vida e promove o bem- } \\
\text { estar emocional a longo prazo. }\end{array}$ & $\begin{array}{l}\text { Hughes; Rogerson; Barton; } \\
\text { Bragg, (2019) }\end{array}$ \\
\hline $\begin{array}{l}\text { O contato com a natureza ajuda a fomentar a criatividade, } \\
\text { iniciativa, autoconfiança, capacidade de escolha e a tomar } \\
\text { decisões e resolver problemas, que por sua vez, contribuem } \\
\text { para o desenvolvimento de múltiplas linguagens e melhora a } \\
\text { coordenação psicomotora. }\end{array}$ & Becker et al (2019) \\
\hline $\begin{array}{l}\text { A conectividade com a natureza favorece a espiritualidade, o } \\
\text { bem-estar pessoal e o comportamento sustentável. }\end{array}$ & Navarro et al (2020). \\
\hline
\end{tabular}

Fonte: elaborado pelos autores.

Ao levar em consideração os indicativos dos diferentes estudos relacionados aos benefícios facultados pelo contato com a natureza, no Quadro 1 , percebe-se que esses benefícios são eficazes aos serem indicados para atenuarem muitos dos males que se expandiram nos últimos anos, e dentre estes, Becker et al. (2019) apontam o distanciamento da natureza; redução das áreas naturais; poluição ambiental; e a falta de segurança e qualidade dos espaços públicos ao ar livre, que nos levam - adultos, jovens e crianças - a passar a maior parte do tempo em ambientes fechados e isolados. Os mesmos autores mencionam que esse cenário traz um ônus elevado para o desenvolvimento saudável das crianças e adolescentes, e para a saúde do planeta. Portanto, ao intensificar o déficit de natureza surgem problemas físicos e mentais em todas as fases do desenvolvimento humano (LOUV, 2016). 
Com o surgimento da COVID-19 muitas incertezas foram evidenciadas, e ao passo que as pessoas foram pressionadas a ficarem em casa, buscou-se no uso de tecnologias digitais respostas para as incertezas, alternativas para continuar trabalhando, atendimento médico e psicológico, entretenimento, acesso a alimentos, entre outros usos possíveis. Segundo Becker et al. (2019), essa imersão no mundo digital, exposição crescente à publicidade e conteúdos tóxicos, violentos ou inadequados, produzem efeitos na saúde física e mental, além da perda de contato com o mundo real e com as relações presenciais, sedentarismo, obesidade e outras consequências que ocasionam intoxicação digital a que todos estamos sujeitos.

Ao considerar que as UCs são constituídas de belas paisagens, rica biodiversidade, e capazes de oportunizar às pessoas uma reconexão com a natureza, enfatiza-se que a visitação nessas áreas pode amenizar os efeitos de ansiedade e o estresse desencadeados, próprios da pandemia da COVID-19. Isso se reforça, também, no que foi mencionado por Maretti et al. (2019), quando sinalizam que presença de UCs contribui significativamente para a qualidade vida das populações, para a sua saúde física e mental, para recreação, além de proporcionar variações agradáveis de temperatura.

Em números, correspondem a 2.446 UCs representadas por distintas categorias distribuídas em todo o território nacional (CNUC, 2020), nos domínios morfoclimáticos brasileiros (Figura 2) e que representam diferentes possibilidades de contato com a natureza.

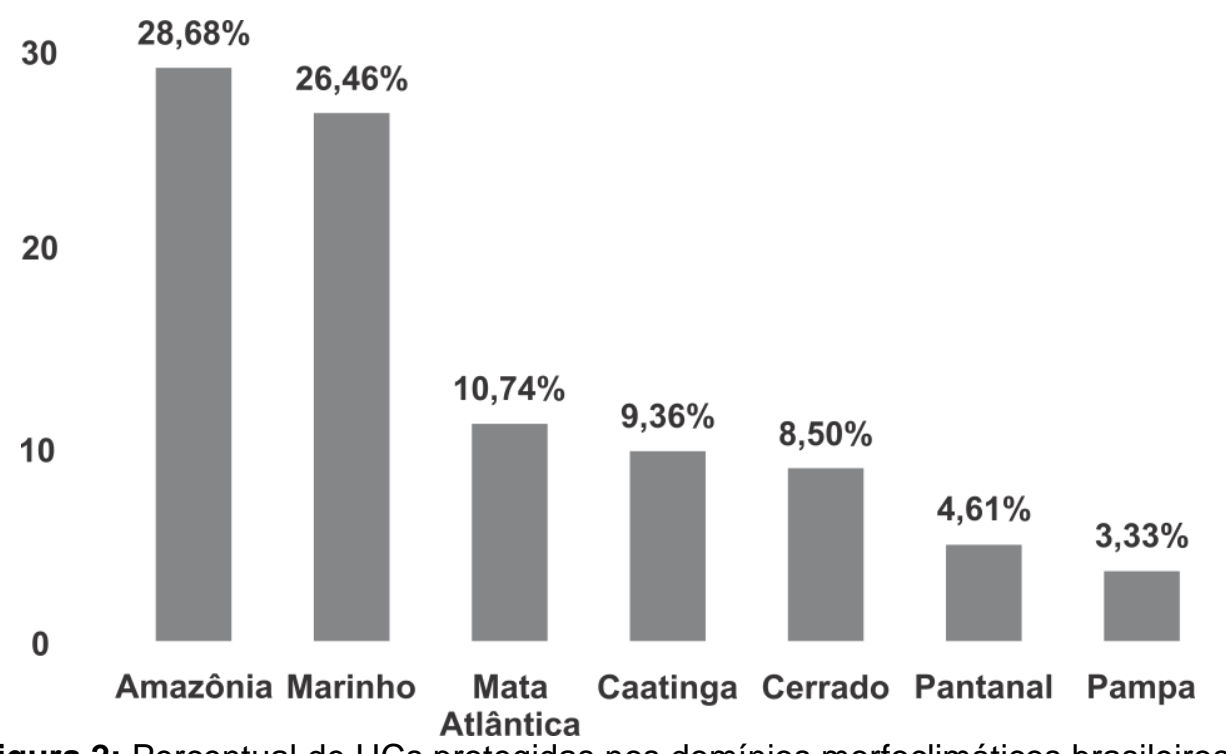

Figura 2: Percentual de UCs protegidas nos domínios morfoclimáticos brasileiros.

Fonte: elaborado com base no CNUC (2020).

Assim, face à distribuição das UCs no território brasileiro, por sua variedade de ecossistemas e diversidade biológica, vale destacar que os benefícios fornecidos pelas Unidades de Conservação para o bem-estar humano podem ser contabilizados em múltiplos efeitos restauradores (Figura $3)$. 


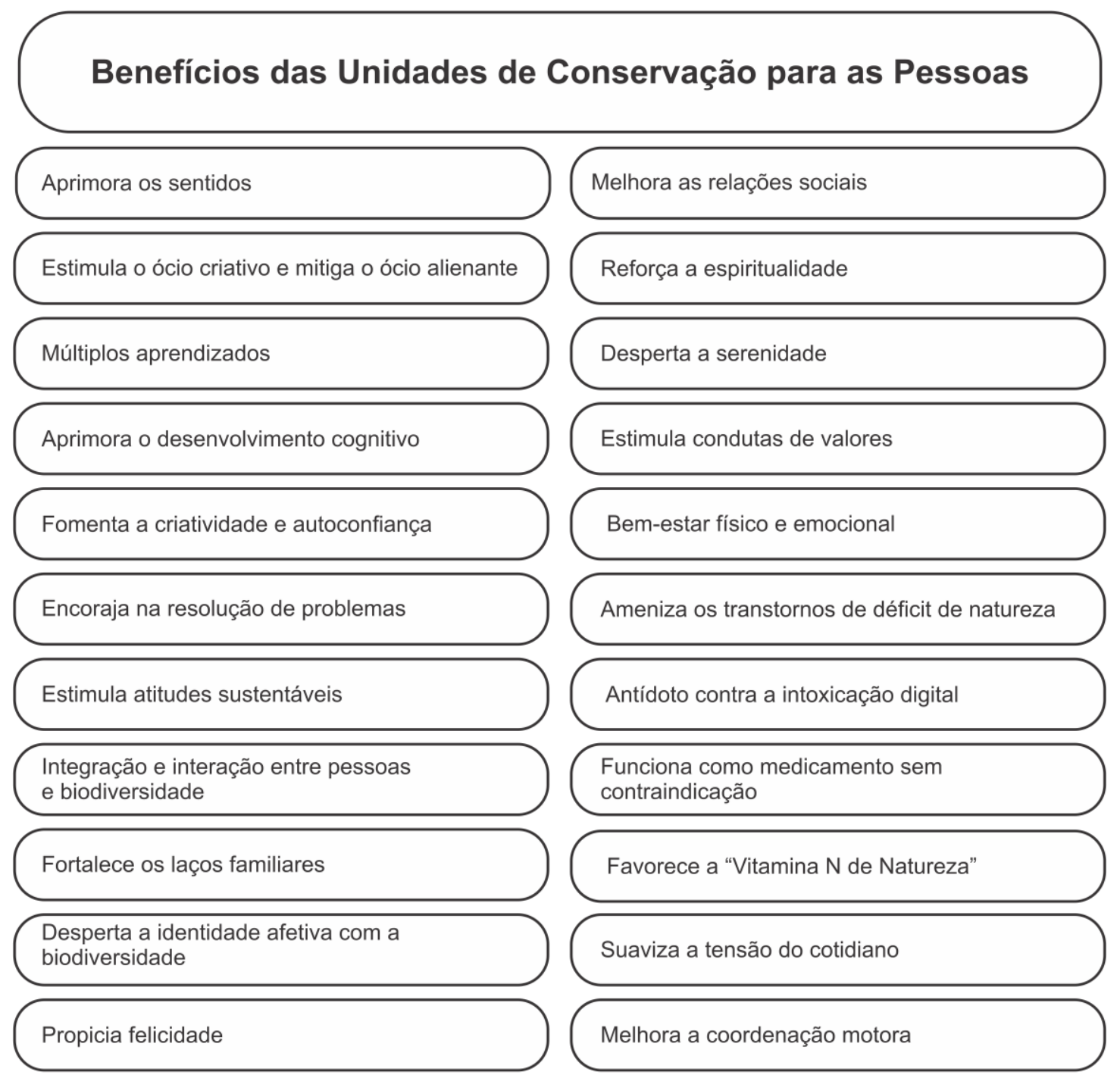

Figura 3: Benefícios das Unidades de Conservação para o bem-estar humano Fonte: elaborado com base em Louv (2016); Richardson; Sheffield (2017); McMahan (2018); Hughes et al. (2019); Becker et al. (2019).

As UCs são áreas-chaves para a contemplação da vida silvestre, lazer e recreação em contato com a natureza, além de serem espaços para atividades de Educação Ambiental que aproximam as pessoas da natureza. Desse modo, a Educação Ambiental pode ser um canal viável na compreensão a respeito do meio ambiente, haja vista que este meio engloba uma rede de relações, não apenas naturais, mas também sociais e culturais (CARVALHO, 2018), como resultantes de experiências restauradoras que provêm da inter-relação dos componentes físicos e sociais, caracterizados por diferentes dimensões afetivas (GRESSLER; GÜNTHER, 2013).

Esse contato com a natureza se caracteriza como um meio de intervenção nas atitudes comportamentais positivas das pessoas, de conexões 
afetivas com a natureza, que proporcionam bem-estar psicológico ao usuário (HARTIG et al.,1997; HINDS; SPARKS, 2008; LOUV, 2016; BERTO et al., 2018; ROSA; PROFICE, 2018; ROSA; COLLADO, 2019), fatores que demonstram que, quando existem relações de proximidade com a natureza, melhora a qualidade de vida e condições de saúde para as pessoas. Por isso, é vital estabelecer conexão com elementos da natureza; e conservá-la faz parte do nosso instinto de sobrevivência (WILSON, 1984), haja vista que a natureza se sustenta perfeitamente sem o ser humano, mas o ser humano não vive sem a natureza, pois, ele é parte e depende exclusivamente dela.

Sendo assim, existem diversas práticas capazes de reconectarem pessoas aos ambientes naturais (Figura 4). Dentre estas, sinalizam:

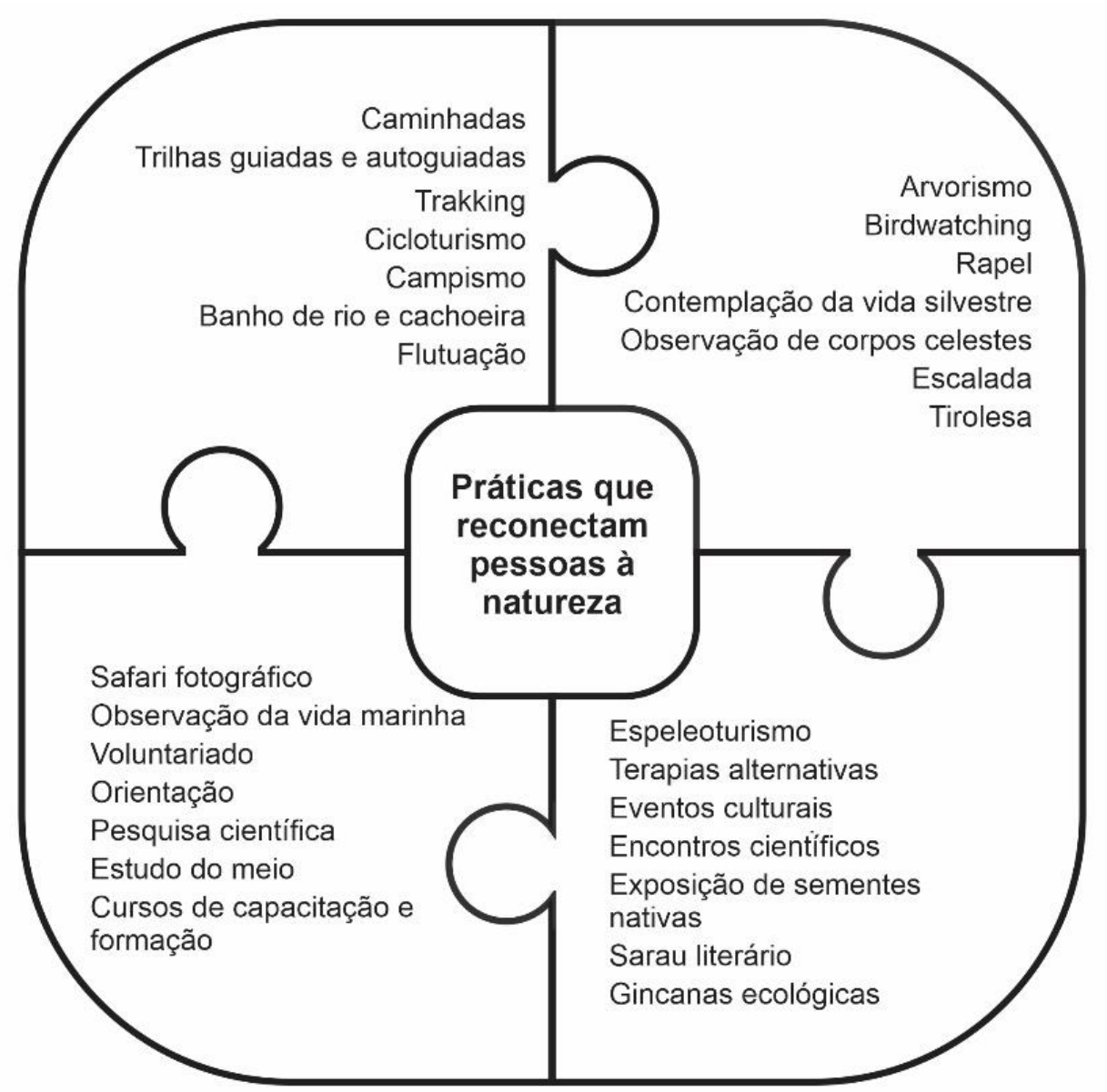

Figura 4: Práticas que reconectam pessoas à natureza.

Fonte: elaborado pelos autores.

São diferentes atividades recreativas, educativas e de interpretação ambiental capazes de reconectarem as pessoas à natureza. Todavia, qualquer que seja a prática, é necessário incorporar ações de Educação Ambiental (WICK; SILVA, 2015). Tendo em vista que apenas a natureza vivida 
diretamente, não contribui para o pleno desenvolvimento psicossomático de uma consciência ambiental e, por isso, reitera a necessidade de atualização das tendências inatas biofílicas, diante da aprendizagem em contexto natural (KELLERT, 2012).

A Educação Ambiental é um componente chave nessa reconexão. Visto que, tem por princípios o enfoque humanista, holístico, democrático e participativo, e possibilita uma compreensão do meio ambiente em sua totalidade alicerçada na sustentabilidade, relações sociais e preocupação ecológica (BRASIL, 1999; CARVALHO, 2018). A Educação Ambiental é apta em viabilizar ações que fomentam vínculos afetivos com a natureza, além de ser um elo importante na conexão promissora entre a saúde humana e as áreas naturais.

As pessoas apresentam necessidades profundas de contato com a natureza (WILSON,1984), e nessa perspectiva, ao aplicar um instrumento que possibilitasse reunir dados para uma melhor compreensão sobre quais os ambientes proporcionam bem-estar, foi constatado que os respondentes do presente estudo, em sua grande maioria optaram por ambientes naturais como espaços a serem frequentados após a pandemia do novo coronavírus e do estabelecimento de um novo "normal" na vida cotidiana.

A fim de compreender as preferências das pessoas em relações aos ambientes, foram agrupadas fotografias que pudessem retratar elementos que compõem os ambientes naturais e artificiais (Figura 5).

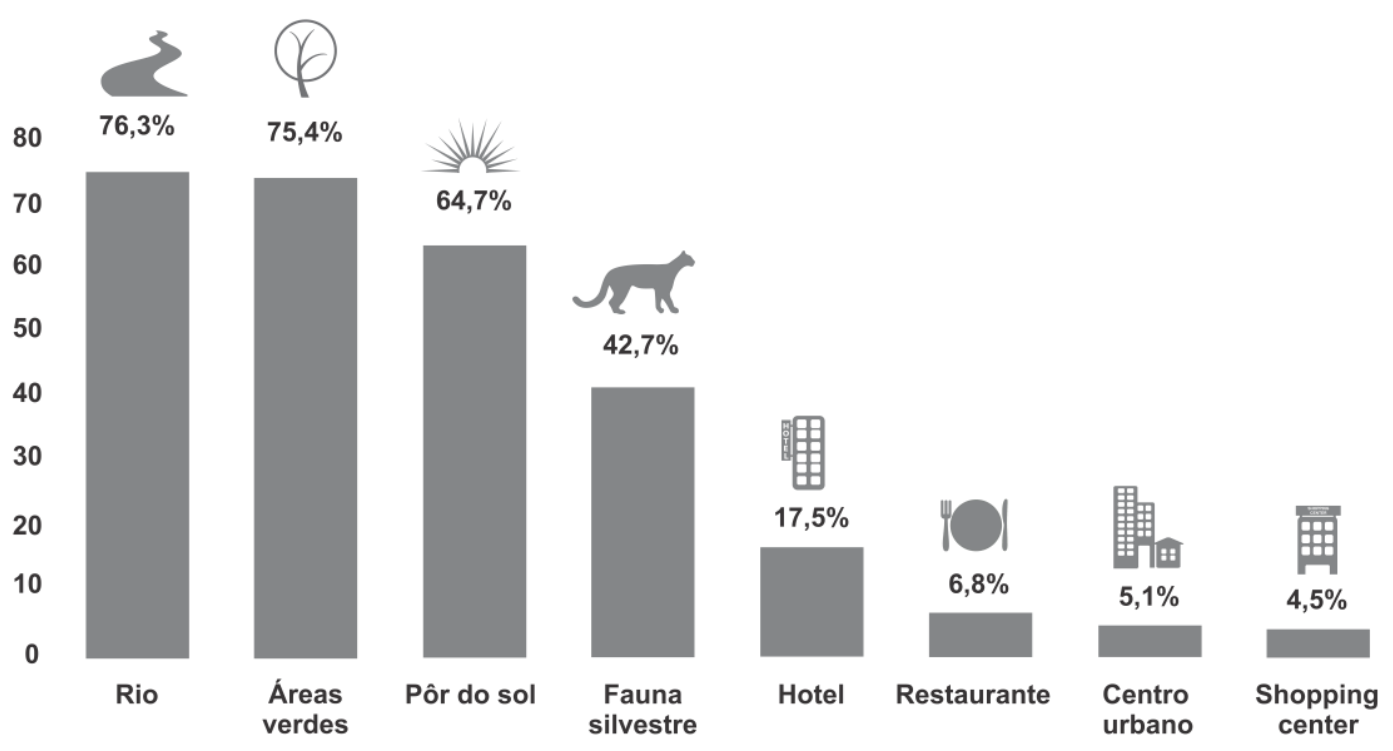

Figura 5: Elementos que compõem os ambientes naturais e artificiais.

Fonte: elaborado pelos autores. 
Os ambientes naturais e seus elementos (paisagens, cachoeiras, montanhas, árvores, aves e outros animais livres na natureza) se destacaram com índice de 83,9\%, como espaços capazes de proporcionarem sensação de bem-estar, após a pandemia da COVID-19. Enquanto o percentual indicado para os ambientes artificiais (shopping centers, lojas, centros comerciais, cafeterias, restaurantes, bares, parques de diversões, dentre outros) foi de $16,1 \%$. Dentre as regiões brasileiras pesquisadas, foram obtidos os seguintes índices: Centro-Oeste - 56,5\%; Nordeste - 17,8\%, Sudeste - 11\%; Sul - 7,6\%; e Norte $-7,1 \%$.

Os índices apresentados corroboram com o que foi apontado por Wilson (1984), na hipótese da biofilia, que as preferências humanas listadas e correspondentes aos elementos da natureza estão relacionadas a uma condição primária e fundamental de nossa espécie.

Além disso, foi identificado que $81,4 \%$ das pessoas conhecem algum tipo de Unidade de Conservação; e pós COVID-19, um índice de 93,8\% pretendem visitar uma dessas áreas. Portanto, isso demonstra que as UCs são espaços apropriados para atenuarem os efeitos de ansiedade e estresse desencadeados pela pandemia da COVID-19, bem como, reconectar pessoas à natureza.

\section{Conclusões}

Em virtude dos benefícios existentes nas Unidades de Conservação, constatou-se que essas áreas são significativamente importantes para o bemestar humano e oportunas para reconexão das pessoas com a natureza, face à eventualidade da pandemia da COVID-19.

Os diversos benefícios sinalizados pela literatura mundial, tendo como temática o contato com a natureza, notabilizam os efeitos restauradores das UCs como espaços possíveis de amenizarem os males causados pela ansiedade e do estresse relacionados à pandemia.

Quanto às práticas capazes de reconectarem pessoas aos ambientes naturais, foi constatada uma variedade de atividades benéficas. No entanto, vale ressaltar que a realização de qualquer atividade deve estar alicerçada nos princípios que regem a Educação Ambiental, entendida como um componentechave nessa reconexão. Portanto, as Unidades de Conservação, compostas por singularidades, representam um guarda-chuva de possibilidades na reconexão de pessoas à natureza. Por essas razões, sugere-se que:

Os gestores das diferentes categorias de Unidades de Conservação do Brasil façam um chamamento à sociedade e divulguem a importância dessas áreas que agregam valores sociais, econômicos e ambientais, além de oportunizarem o uso público e o turismo sustentável.

As Instituições educacionais, tanto da esfera pública, quanto da iniciativa 
Aos diferentes atores do turismo que planejam atividades nas UCs, orienta-se vincular ações de Educação Ambiental adequadas aos objetivos de criação da área.

Para a sociedade, de modo geral, ao desejar saúde física e mental, visite uma Unidade de Conservação e estabeleça uma conexão com a natureza.

\section{Agradecimentos}

Ao Programa de Pós-Graduação em Meio Ambiente e Desenvolvimento Regional da Anhanguera-Uniderp; à Coordenação de Aperfeiçoamento de Pessoal de Nível Superior (CAPES), visto que o presente trabalho foi realizado com apoio da concessão de bolsa de estudos.

\section{Referências}

BRASIL. Lei no 9.795, de 27 de abril de 1999. Dispõe sobre a Educação Ambiental, institui a Política Nacional de Educação Ambiental e dá outras providências. Brasília, DF: Senado, 1999. Disponível em: <http://www.planalto.gov.br/ccivil 03/leis/19795.htm>. Acesso em: 13 jun. 2020.

BECKER, D.; SOLÉ, D.; TING, E.; EISENSTEIN, E.; MARTINS FILHO, J.; FLEURY, L.; SILVA, L. R.; BARROS, M. I A.; GHELMAN, R.; WEFFORT, V. R. $S$. Benefícios da Natureza no Desenvolvimento de Crianças e Adolescentes. Manual de Orientação. São Paulo: Instituto Alana e Sociedade Brasileira de Pediatria, 2019. Disponível em: $<$ https://www.sbp.com.br/fileadmin/user upload/manual orientacao sbp cen . pdf>. Acesso em: 10 maio 2020.

BERTO, R.; BARBIERO, G.; BARBIERO, P.; SENES, G. An individual's connection to nature can affect perceived restorativeness of natural environments. Some observations about biophilia. Behavioral Sciences, v. 8, n.3, p. 34, 2018.

BRATMAN, G. N.; HAMILTON, J. P.; HAHN, K. S.; DAILY, G. C.; GROSS, J. J. Nature experience reduces rumination and subgenual prefrontal cortex activation. Proceedings of the national academy of sciences, v. 112, n. 28, p. 8567-8572, 2015.

BRÜGGER, A.; KAISER, F. G.; ROCZEN, N. Connectedness to nature, inclusion of nature, environmental identity, and implicit association with nature. European Psychologist, v. 16, p. 324- 333, 2011.

CARVALHO, I. C. M. Educação Ambiental: a formação do sujeito ecológico. São Paulo: Cortez, 2018. 
CNUC. Cadastro Nacional de Unidades de Conservação. Painel das Unidades de Conservação Brasileiras. Disponível em: <http://www.mma.gov.br/areasprotegidas/cadastro-nacional-de-ucs>. Acesso em: 10 jun. 2020.

FIGGIS, P.; MACKEY, B.; FITZSIMONS, J.; IRVING, J.; CLARKE, P. Valuing nature: protected areas and ecosystem services. Australian Committee for IUCN: Sydney, NSW, 2015.

FUREDI, F. Para uma sociologia do medo. In: MENDES, J. M. O. (Coord.). Risco, cidadania e Estado num mundo globalizado. Coimbra: Contexto, CES - Centro de Estudos Sociais, 2013, p. 191-210.

GRESSLER, S. C.; GÜNTHER, I. A. Ambientes restauradores: definição, histórico, abordagens e pesquisas. Estudos de Psicologia, v. 18, n. 3, p. 487495, 2013.

HARTIG, T.; KORPELA, K.; EVANS, G. W.; GÄRLING, T. A measure of restorative quality in environments. Scandinavian housing and planning research, v. 14, n. 4, p. 175-194, 1997.

HINDS, J.; SPARKS, P. Engaging with the natural environment: The role of affective connection and identity. Journal of environmental psychology, $v$. 28, n. 2, p. 109-120, 2008.

HO, C. SH; CHEE, C. Yi; HO, R. Cm. Mental health strategies to combat the psychological impact of COVID-19 beyond paranoia and panic. Ann Acad Med, v. 49 , n. 1 , p. 1-3, 2020.

HUGHES, J.; ROGERSON, M.; BARTON, J.; BRAGG, R. Age and connection to nature: when is engagement critical? Frontiers in Ecology and the Environment, v.17, n. 5, p. 265-269, 2019.

KELLERT, S. Birthright: People and Nature in the Modern World. Yale University Press, 2012.

KENIGER, L. E.; GASTON, K. J; IRVINE, K.N; FULLER, R. A. What are the Benefits of Interacting with Nature? International Journal of Environmental Research and Public Health, Gland, v.10, p.913-935, 2013.

LANZAS, M.; HERMOSO, V.; DE-MIGUEL, S.; BOTA, G.; BROTONS, L. Designing a network of green infrastructure to enhance the conservation value of protected areas and maintain ecosystem services. Science of the Total Environment, v. 651, p. 541-550, 2019. Acesso em: 19 maio 2020.

LOUV, R. Vitamin N: The essential guide to a nature-rich life. Algonquin Books, 2016.

LOUV, R. A última criança na natureza: resgatando nossas crianças do transtorno do deficit de natureza. São Paulo: Aquariana; 2016. 
MACÊDO, F. D. O. A.; LOPES, K. A. P; LOPES, L. A. M. R.; DE FRANÇA CRUZ, R. Ações e experiências de terapeutas ocupacionais no contexto de pandemia da COVID-19/Occupational Therapists actions and expiriences in the COVID-19 pandemic context. Revista Interinstitucional Brasileira de Terapia Ocupacional-REVISBRATO, v. 4, n. 3, p. 318-333, 2020.

MARTINS, H. Humanos e não-humanos em ambientes partilhados: Notas introdutórias a uma antropologia das áreas protegidas. Análise Social, v. 1, n. 226, pp. 28-56, 2018.

MARETTI, C. C.; VON BEHR, M.; SOUZA, T. V. B.; MATOS SCARAMUZZA, C. A.; GUIMARÃES, E.; ELIAS, P. F.; DE BRITO, M. C. W. Ciudades y áreas protegidas en Brasil: Soluciones para el bienestar, la conservación de la naturaleza y la participación activa de la Sociedad. In: GUERRERO, E, F. Voces Ciudades Sostenibles y Resilientes. Bogotá: Ministério de Ambiente y Desarrollo Sostenible, 2019.

MCMAHAN, E. A. Happiness comes naturally: Engagement with nature as a route to positive subjective well-being. In: DIENER, E.; OISHI, S.; TAY, L. (Eds.), Handbook of well-being. Salt Lake City, UT: DEF Publishers, 2018.

MOGHADAM, D. M; SINGH, H. J; YAHYA, W. R. W. A Brief Discussion on Human/Nature Relationship. International Journal of Humanities and Social Science, v. 5, n. 6, p. 90-93, 2015

NAVARRO, O.; TAPIA-FONLLEM, C.; FRAIJO-SING, B.; ROUSSIAU, N.; ORTIZ-VALDEZ, A.; GUILLARD, M.; FLEURY-BAHI, G. Connectedness to nature and its relationship with spirituality, wellbeing and sustainable behaviour (Conectividad con la naturaleza y su relación con la espiritualidad, el bienestar y la conducta sustentable). Psyecology, v.11, n. 1, p. 37-48, 2020.

PANCANI, L.; MARINUCCI, M.; AURELI, N.; RIVA, P. Forced social isolation and mental health: A study on 1006 Italians under COVID-19 quarantine. PsyArXiv, p. 1-21, 2020.

RAJKUMAR, R. P. COVID-19 and mental health: A review of the existing literature. Asian journal of psychiatry, p.102066, 2020. Disponível em: $<$ https://www.ncbi.nlm.nih.gov/pmc/articles/PMC7151415/>. Acesso em: 12 maio 2020.

RICHARDSON, M.; SHEFFIELD, D. Three good things in nature: Noticing nearby nature brings sustained increases in connection with nature/Tres cosas buenas de la naturaleza: Prestar atención a la naturaleza cercana produce incrementos prolongados en conexión con la naturaleza. Psyecology, v. 8, n. 1, p. 1-32, 2017. >. Acesso em: 12 maio 2020.

ROSA, C. D.; COLLADO, S. Experiences in nature and environmental attitudes and behaviors: Setting the ground for future research. Frontiers in psychology, v. 10, p. 763, 2019. 
ROSA, C. D.; PROFICE, C. C. Que tipo de Educação Ambiental e para quem? Fatores associados a atitudes e comportamentos ambientais. Revista Brasileira de Educação Ambiental (RevBEA), v. 13, n. 1, p. 111-125, 2018.

SCHERTZ, K. E.; BERMAN, M. G. Understanding nature and its cognitive benefits. Current Directions in Psychological Science, v. 28, n. 5, p. 496502, 2019.

ULRICH, R. S. Aesthetic and affective response to natural environment. In: ALTMAN, I.; WOHLWILL, J. F. (Orgs.), Behavior and the Natural Environment, v. 06, p. 85-120, Nova lorque: Plenum, 1983.

VAN DEN BERG, A. E.; JOYE, Y.; DE VRIES, S. Health benefits of nature. In STEG, L.; VAN DEN BERG, A E.; DE GROOT, J. I. M. (Eds.), Environmental psychology: An introduction. Oxford: BPS Blackwell, 2012. p. 47-56.

WICK, M. A. L.; SILVA, L. F. Unidades de Conservação e processos em Educação Ambiental. Revista Brasileira de Educação Ambiental (RevBEA), v. 10, n. 1, p. 201-220, 2015.

WILSON, E. O. Biophilia. Boston: Harvard University Press, 1984. 157p. 